\title{
Modélisation de l'expansion hydrodynamique d'un panache d'ablation laser
}

\author{
M.L. Sentis, H.C. Le, W. Marine, J.D. Parisse et D. Zeitoun \\ Groupe Interdisciplinaire Ablation Laser et Applications, IRPHE, UMR 6594, GPEC, UMR 6631, \\ IUSTI, UMR 6595, Campus de Luminy, Case 918, 13288 Marseille cedex 9, France
}

\begin{abstract}
Résumé : L'étude présentée porte sur la simulation 2-D de l'expansion d'un plasma induit par ablation laser UV sous atmosphère neutre. L'écoulement bidimensionnel, axisymètrique, instationnaire, dissipatif et ionisé est étudié par la résolution des équations de Navier-Stokes. L'ablation laser étudiée est celle d'un matériau simple, le silicium (Si) sous atmosphère d'argon ou d'hélium. L'écoulement est constitué de quatre espèces monoatomiques $\mathrm{Si}, \mathrm{Si}+$, électrons et $\mathrm{Ar} / \mathrm{He}$. L'ionisation est considérée hors équilibre et caractérisée par des processus tels que l'ionisation par impact électronique, la recombinaison à trois corps et la recombinaison radiative.
\end{abstract}

\section{INTRODUCTION}

Le procédé d'ablation à l'aide d'un laser impulsionnel de matériaux divers est employé dans un grand nombre d'applications telles que : micro-usinage, traitements et nettoyage de surface, dépôts de couches minces, réalisation de nano-agrégats, etc. Les processus physiques intervenant lors de l'ablation laser sont souvent complexes et encore mal compris. L'amélioration de nos connaissances notamment dans les domaines de l'hydrodynamique et de la cinétique des plasmas passe bien entendu par l'étude expérimentale des processus d'ablation mais aussi par le développement de modèles numériques performants. Depuis le début des années 90, un certain nombre de modèles sont développés [1-6]. Ces modèles sont encore relativement simples, souvent $1 \mathrm{D}$ et basés sur les équations d'Euler qui ne permettent pas de traiter les phénomènes de transport (diffusion, flux de chaleur, effets visqueux). Le développement de modèles $2 \mathrm{D}$ prenant en compte à la fois les deux étapes successives et interdépendantes d'interaction laser-matière et du transport des espèces avec les effets dissipatifs est donc nécessaire. Dans le présent travail, un model $2 \mathrm{D}$ basé sur la résolution des équations de Navier-Stokes permet l'étude d'un écoulement bidimensionnel, axisymètrique, instationnaire, dissipatif et ionisé.

\section{MODELE NUMERIQUE}

\subsection{Hypothèses}

Dans ce modèle, on étudie l'irradiation d'une cible en silicium par un laser ArF ( $\lambda=193 \mathrm{~nm}$ ) d'une durée d'impulsion de $15 \mathrm{~ns}$ et pour une densité d'énergie surfacique comprise entre 1,5 et $2,4 \mathrm{~J} / \mathrm{cm}^{2}$. Quatre espèces sont prises en compte : Sil, SilI, électrons, et les atomes de gaz neutre (ArI ou HeI). Le panache d'ablation ainsi que le gaz environnant sont considérés comme un fluide compressible $[1,6]$. Dans le plasma, le critère de quasi-neutralité est respecté, et deux températures ( $T$ et $T_{e}$ ) sont utilisées pour décrire l'ionisation hors équilibre $[6,7]$.

\subsection{Equations générales}

Les équations de Navier-Stokes sont donc résolues dans le cas d'un gaz parfait compressible muti-espèces en prenant compte des phénomènes de diffusion, de conduction thermique ainsi que les effets de viscosité. Les équations de conservation (masse $\rho_{i}$, quantité de mouvement $\rho \vec{v}$, énergie des particules lourdes $E_{a}$ et des électrons $E_{e}$ ) pour les quatre espèces i, sont les suivantes :

$$
\frac{\partial \rho_{i}}{\partial t}+\operatorname{div}\left(\rho_{i}\left(\vec{v}+\vec{r}_{d i}\right)\right)=\omega_{i}, \quad i=1, \ldots, 4
$$




$$
\begin{aligned}
& \frac{\partial \rho \vec{v}}{\partial t}+\operatorname{div}(\rho \vec{v} \vec{v}-\bar{\sigma})=0 \\
& \frac{\partial E_{a}}{\partial t}+\operatorname{div}\left(E_{a} \vec{v}-\bar{\sigma} \vec{v}+\vec{q}\right)=\omega_{a} \\
& \frac{\partial E_{e}}{\partial t}+\operatorname{div}\left(\left(E_{e}+P_{e}\right)\left(\vec{v}+\vec{v}_{d i e}\right)\right)=\omega_{e}+F_{e}
\end{aligned}
$$

où, $\vec{v}$ est la vitesse, $\vec{v}_{d i}$ la vitesse de diffusion, $\omega_{i}$ le terme source de production de l'espèce $i, \omega_{a}$ le terme source de transfert d'énergie entre particules et électrons, $\omega_{e}$ le terme source de transfert d'énergie au cours des collisions élastiques et inélastiques, $\overline{\bar{\sigma}}$ le tenseur de contraintes, $\vec{q}$ le flux de chaleur, $P_{e}$ la pression électronique, et $F_{e}$ la force induite par le champ électrique.

Les équations complémentaires concernant le tenseur de contraintes $\overline{\bar{\sigma}}$ et la densité $\rho$ du mélange sont :

$$
\overline{\bar{\sigma}}=-P_{a} \overline{\bar{I}}+\bar{\tau} \text { and } \rho=\sum_{i} \rho_{i}
$$

où, $\overline{\bar{\tau}}$ est le tenseur des contraintes visqueuses et $P_{a}$ la pression hydrostatique.

Si on considère que chaque espèce suit la loi des gaz parfaits, alors la pression hydrostatique $P_{a}$ peut s'écrire sous la forme $P_{a}=\sum_{i} \rho_{i} R T / \hat{M}$, où $\hat{M}$ est la masse molaire du mélange, et $R$ la constante universelle des gaz. La pression électronique peut être déterminée à partir de $P_{e}=\rho_{e} R T_{e} / \hat{M}_{e}$ où $\rho_{e}$ et $\hat{M}_{e}$ sont respectivement la densité et la masse molaire des électrons.

Le flux de chaleur $\vec{q}$ peut se mettre sous la forme $\vec{q}=-\lambda_{m} \nabla T+\rho \sum_{i} \vec{v}_{d_{i}} h_{i} y_{i}$ où, $\lambda_{m}$ est la conductivité thermique du mélange gazeux, et $h_{i}$ l'enthalpie déterminée par $h_{i}=\int_{0}^{T} C_{p_{i}} d T+h_{i_{\dot{\alpha}}}$ avec $C_{p_{i}}$ et $h_{i_{a}}$ représentant la chaleur spécifique à pression constante et l'enthalpie de formation de l'espèce considérée. L'expression rigoureuse de la vitesse de diffusion telle qu'elle peut être donnée par Hirschfelder et al. [8] est difficile à intégrer dans un modèle numérique. Oran \& Boris donnent des expressions simplifiées qui permettent d'évaluer la vitesse de diffusion et les coefficients de transport [9]. Les vitesses de diffusion sont les solutions du système d'équations suivant : $\overrightarrow{\operatorname{grad}} X_{i}=\sum_{j} \frac{X_{i} Y_{j}}{D_{i j}}\left(\vec{v}_{d j}-\vec{v}_{d i}\right)$

où, pour chaque espèce $\mathrm{i}, X_{i}$ est la fraction molaire, $Y_{i}$ la fraction massique et $D_{i j}$ le coefficient de diffusion de l'espèce $i$ dans l'espèce $j$. On considère dans ce modèle que la contribution des diffusions thermique et de pression est négligeable par rapport à celle de la diffusion massique.

La diffusion induite dans l'écoulement par la présence d'un champ électrique doit aussi être prise en compte. En effet, les électrons ont une vitesse de déplacement plus rapide que celle des ions, laissant derrière eux un excès de charges positives. Le champ électrique induit par cette séparation de charges initiale tend alors à ralentir les électrons et à accélérer les ions et ainsi affecte la diffusion entre électrons et ions. Le champ électrique agit également sur les particules chargées et peut changér de manière significative leur énergie cinétique. La prise en compte de ce phénomène de diffusion induit par la présence du champ électrique demande la résolution d'un système d'équations très complexe [10]. Dans le modèle, ce phénomène est approximé en introduisant le concept de diffusion ambipolaire dans lequel la séparation de charges est faible et où tout courant électrique est nul [10].

La force induite par le champ électrique intervenant dans l'équation (4) peut se calculer à partir de l'expression suivante $[7,10]: F_{e}=\overrightarrow{g r a d} P_{e}\left(\vec{v}+\vec{v}_{d i e}\right)$

Pour la conductivité thermique du mélange, $\lambda_{m}$, on peut utiliser une équation donnée par Mason et Saxena [11] et plus récemment par Oran \& Boris [9]: 


$$
\lambda_{m}=\sum_{i} \lambda_{i}\left[1+\frac{1.065}{2 \sqrt{2 n_{i}}} \sum_{k \neq i} n_{k} \Phi_{i k}\right]^{-1} \text { avec } \Phi_{i k}=\frac{\left[1+\left(\lambda_{i} / \lambda_{k}\right)^{1 / 2}\left(\hat{M}_{i} / \hat{M}_{k}\right)^{1 / 4}\right]^{2}}{\left[1+\hat{M}_{i} / \hat{M}_{k}\right]^{1 / 2}}
$$

$\lambda_{i}$ étant la conductivité thermique de l'espèce i, $\hat{M}_{i}$ et $\hat{M}_{k}$ la masse molaire des espèces $i$ et $k$.

La viscosité du mélange est donnée par [12]: $\mu_{m}=\sum_{i} \mu_{i}\left[1+\frac{\sqrt{2}}{4 n_{i}} \sum_{k \neq i} n_{k} \varphi_{i k}\right]^{-1}$

où, $\mu_{i}$ est la viscosité de l'espèce $i_{2}$, et $\varphi_{i k} s^{2}$ exprimant comme :

$$
\varphi_{i k}=\frac{\left[1+\left(\mu_{i} / \mu_{k}\right)^{1 / 2}\left(\hat{M}_{i} / \hat{M}_{k}\right)^{1 / 4}\right]^{2}}{\left[1+\hat{M}_{i} / \hat{M}_{k}\right]^{1 / 2}}
$$

Une description détaillée des coefficients de transport donnés sous forme de fonctions polynomiales de la température peut se trouver dans [13].

\subsection{Ionisation hors équilibre}

Les termes sources $\omega_{i}, \omega_{a}$, et $\omega_{e}$ des équations (1), (3) et (4) dépendent des réactions d'ionisation et de recombinaison prenant place dans le panache d'ablation. Dans ce modèle les réactions suivantes sont prises en compte :

$$
\begin{aligned}
& \mathrm{SiI}+\mathrm{e}<--->\mathrm{SiII}+\mathrm{e}+\mathrm{e} \\
& \mathrm{SiII}+\mathrm{e}<--->\mathrm{SiI}+\mathrm{hv}
\end{aligned}
$$

Le taux d'ionisation par impact électronique, $C_{1}$, de la réaction (11) est déterminé à partir de la relation suivante [6]: $C_{1}=2 \frac{\left(2 \pi m_{e} k T_{e}\right)^{3 / 2}}{n_{e} h^{3}} C_{2} \exp \left(\frac{-U_{i}}{T_{e}}\right)$

où, $m_{e}$ est la masse d'un électron, $U_{i}$ le potentiel d'ionisation de SiI, et $k$ la constante de Boltzman. $C_{2}$ correspond au taux de recombinaison à trois corps déterminé par:

$$
C_{2}=\frac{4 \pi(2 \pi)^{1 / 2}}{9} \times \frac{e^{10}}{m_{e}^{1 / 2}\left(k T_{e}\right)^{9 / 2}} n_{e}
$$

où, $e$ est la charge d'un électron. Le taux de recombinaison radiative $C_{3}$ se calcule à partir de l'expression suivante, dans laquelle $T_{e}$ est exprimée en électron-volts : $C_{3}=2.10^{-13} / \sqrt{T_{e}}$

Le terme source d'énergie de l'équation (4), résultat des collisions élastiques entre atomes neutres et ionisés peut s'écrire sous la forme suivante [13]: $\omega_{a}=3 R \rho_{e}\left(T_{e}-T\right) \sqrt{\frac{8 R T_{e}}{\pi \hat{M}_{e}}} \sum_{i \neq e} \frac{\rho_{e} n_{i}}{\hat{M}_{i}^{2}} \sigma_{e i}$

avec $\sigma_{e i}$ la section efficace de collision entre électrons et l'espèce $\mathrm{i}$.

Le terme source $\omega_{e}$ dans l'équation (4) relatif à l'énergie perdue ou gagnée par les électrons lors de collisions élastiques ou inélastiques est déterminé à partir de $[6,7]$ :

$$
\omega_{e}=-\omega_{a}+2 / 3\left(E^{*} n_{\text {ion }} C_{2}-U_{i} n_{\text {atom }} C_{1}-3 / 2 k T_{e} C_{3}\right)
$$

où, $E^{*}$ est l'énergie gagnée par l'électron au cours des processus de recombinaison à trois corps. Cette énergie peut se calculer à partir des valeurs de la densité et de la température $[6,7], n_{\text {atom }}$ et $n_{i o n}$ sont les densités respectives des atomes neutres et ionisés.

\subsection{Méthode numérique}

Les équations (1) à (4) sont résolues pour les flux convectifs (flux Eulériens) au moyen de l'algorithme LCPFCT développé en 1987 par Oran et al. [9] et récemment mis à jour [14]. Cet algorithme explicite est basé sur un schéma de différence finie associé à des méthodes plus générales de correction de flux FCT (Flux Corrected Transport). Les flux diffusifs (viscosité, diffusion, conductivité thermique) sont résolus au moyen d'un schéma de différence finie centrée [15]. 


\section{RESULTATS}

La figure 1 présente, comme exemple, l'évolution temporelle de la température des atomes (a) et de la vitesse axiale des particules (b) pour une densité de puissance laser de $170 \mathrm{MW} / \mathrm{cm}^{2}$ et une pression d'argon de 400 mTorr. Les vitesses négatives (fig. 1b) traduisent un reflux du panache vers la cible, phénomène observé expérimentalement pour la première fois très récemment [16].

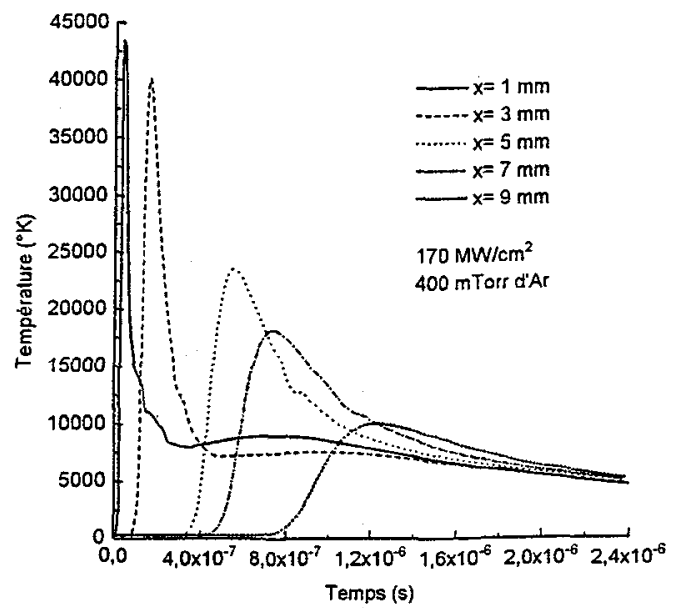

(a)

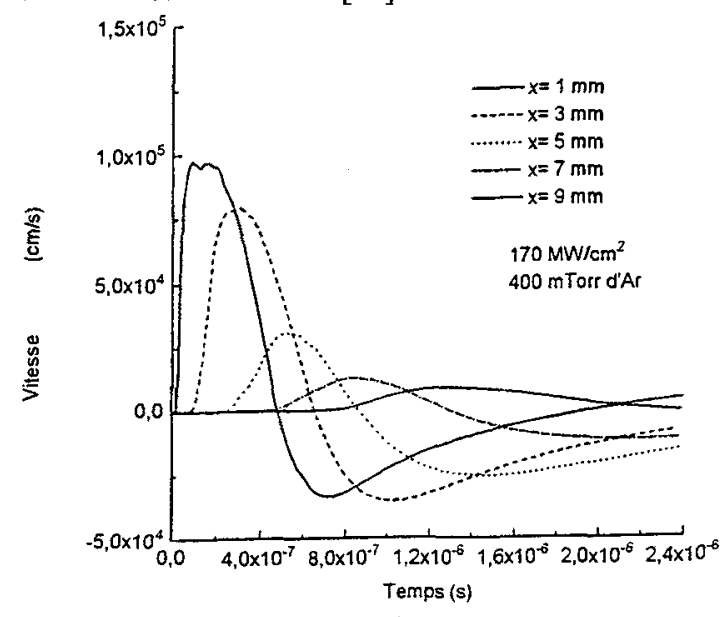

(b)

Figure 1 : Evolution temporelle de la température des atomes (a) et de leur vitesse axiale (b)à différentes distances de la cible.

\section{CONCLUSION}

Le model numérique présenté permet la simulation bidimentionnelle du panache d'ablation sous atmosphère neutre. Cette simulation présente l'originalité d'inclure les phénomènes de diffusion et d'ionisation hors équilibre. Le champ électrique présent dans le panache est également pris en compte de manière simplifiée en introduisant le concept de diffusion ambiplolaire. L'ensemble des résultats issus de ce modèle peuvent se trouver dans $[17,18]$.

\section{Références}

1. R.K. Singh, J. Narayan, Phys. Rev. B 41, 8843 (1990).

2. S.I. Anisimov, D. Bauerle, B. Luk'yanchuk, Phys. Rev. B 48, 12076 (1993).

3. A. Verts, R.W. Dreyfus, D.E. Platt, IBM J. Res. Develop 38, 3 (1994)

4. J.N. Leboeuf, et al. Phys. Plasmas 3, 2203 (1996).

5. H. Le, J. Vuillon, D. Zeitoun, W. Marine, M. Sentis, R.W. Dreyfus, App. Surf. Sci. 96-98, 76 (1996).

6. A.V. Bulgakov, N.M. Bulgakova, J. Phys. D : App. Phys. 28, 1710 (1995).

7. Ya.B. Zel'dovich, Yu.P. Raizer, in Physics of Shock Waves and High-Tempertaure Hydrodynamic Phenomena (Academic Press, New York, 1966).

8. C.F. Curtiss, J.O. Hirschfelder, J. Chemical Phys. 17, 550 (1949).

9. E. Oran, J.P. Boris, in Numerical simulation of reactive flow (Elsevier, New York, 1987).

10. J.H. Lee, in Progress in Astronautics and Aeronautics: Thermal Designed of Aeroassisted Orbital Transfert Vehicules, $96: 3-53$ (1985).

11. E.A. Mason, S.C. Saxena, Physics of Fluids 1, 631 (1958).

12. C.R. Wilke, J. Chem. Phys. 18, 517 (1950).

13. R.J. Kee, G. Dixon-Lewis, J. Warnatz, M. Coltrin, J. Miller, Sandia Report No SAND 86-8246 (1986).

14. J.R. Boris, F.F. Grinstein, E.S. Oran, T.A. Brun, NRL memorandum report N 6410-93-7192 (1993).

15. G. Patnaik, K. Laskey, K. Kailasanath, E.S. Oran, T.A. Brun, NRL memurandum report 6555 (1989).

16. D. Geohegan, A. Puretzky, G. Duscer, S. Pennicook, Appl. Phys. Letter 72, 2987 (1998).

17. H.C. Le, Thèse de l'Université de Provence, Marseille 17 Juin 1998.

18. H.C. Le, D. Zeitoun, J.D. Parisse, M.L. Sentis, W. Marine, en préparation pour Phys. Rev. E. 